ORIGINAL PAPER

Eur. J. Histochem.

(C) Luigi Ponzio e figlio - Editori in Pavia

\title{
Chorion laeve trophoblasts of preeclamptic fetal membranes: histochemically detectable enzyme activities do not change at a subcellular level
}

\author{
S. Matsubara, T. Takayama, R. Iwasaki, A. Izumi, T. Watanabe, and I. Sato
}

Department of Obstetrics and Gynecology, Jichi Medical School, Minamikawachi-machi 3311-1, Kawachi-gun, Tochigi 329-0498, Japan

Accepted: $18 / 5 / 01$

Key words: trophoblast, fetal membrane, ADP-degrading activity, cytochrome c oxidase, preeclampsia

\section{SUMMARY}

We examined the subcellular localization of ADPdegrading activity and cytochrome $c$ oxidase (CCO) activity in chorion laeve trophoblasts from term and near term human fetal membranes, and compared them with those from severe preeclamptic fetal membranes. The methods used for the detection of enzyme activities were the lead nitrate method for ADP-degrading activity and the diaminobenzidine method for CCO. Precipitates indicative of ADP-degrading activity were visible on surface microvillous plasma membranes of chorion laeve trophoblasts both from normal and preeclamptic fetal membranes. The intensity and distribution patterns were the same in the normal and preeclamptic subjects. CCO labeling was visible in almost all laeve trophoblastic mitochondria both in normal and preeclamptic cases. Previously, we demonstrated that in preeclamptic villous trophoblasts there were decreases in ADP-degrading activity and the presence of CCO-negative mitochondria, which were proposed to lead to dysfunction of each villous trophoblast, and finally to placental insufficiency in preeclampsia. Reductions or changes in enzyme intensities/distribution patterns, which are characteristic features of preeclamptic villous trophoblasts, were absent in chorion laeve trophoblasts in preeclampsia. These results suggest that in preeclampsia there are no, or at least less severe, abnormalities in the enzyme activities of chorion laeve trophoblasts, compared with villous trophoblasts, as far as enzyme-histochemically detectable enzymes are concerned.

\section{INTRODUCTION}

Preeclampsia is one of the major unsolved problems in feto-maternal medicine, and is a leading cause of placental insufficiency. It affects approximately $5 \%$ of all pregnancies, and frequently causes fetal/neonatal/maternal morbidity and mortality (Cunningham et al. 1997). This disease occurs not only in pregnant women but also women with hydatidiform moles (abnormal placental proliferation without a fetus), and it disappears immediately after delivery, indicating that preeclampsia is a "placental disease" (Redman 1991; Widschwendter et al., 1998).

Though the etiology and pathogenesis of preeclampsia are still obscure, our previous enzymehistochemical observations demonstrated that, in villous trophoblasts with preeclampsia, some impor- 
tant placental enzymes, such as ADP-degrading enzyme (Matsubara et al. 1997a) and cytochrome c oxidase (CCO) (Matsubara et al. 1997b), exhibited decrease/change in their enzyme intensities or distribution patterns, compared to those without preeclampsia. We proposed that in preeclamptic placenta there were some trophoblastic dysfunctions at each cell level, which may lead to placental insufficiency of preeclampsia. Preeclampsia, therefore, is possibly a "villous trophoblastic cell disease" (Matsubara et al. 1997a,b).

Besides these villous trophoblasts (syncytiotrophoblasts and cytotrophoblasts) in the placenta, there are chorion laeve (cyto)trophoblasts in the fetal membranes (the chorioamnion) (Benirschke and Kaufmann 2000). These two trophoblasts originate from the same stem cells (outer cell mass of the blastocyst) in early human placental/chorioamnion development. Later, trophoblasts located near the implantation pole become villous trophoblasts, while those localized in the anti-implantation pole become chorion laeve trophoblasts (Benirschke and Kaufmann 2000). While the former villous trophoblasts, especially villous syncytiotrophoblasts, were considered to be functioning cells of the placenta and many histochemical observations were made, little attention was paid to chorion laeve trophoblasts. This was due to the belief that chorion laeve trophoblasts may be a less active remnant of the initial capsular chorion, contributing as a mere covering of the amniotic cavity, and thus having lower biological significance. Recent reports, however, disproved this idea. Chorion laeve trophoblasts possessed many enzymes similar to villous trophoblasts (Matsubara et al. 1994; 1997c; Matsubara and Sato 2000), and produced a variety of substances essential for the maintenance of pregnancy, including renin (Poisner et al. 1981), endopeptidases (Germine et al. 1994) and 15-hydroxyprostaglandin dehydrogenase (Sangha et al. 1994), which indicated that chorion laeve trophoblasts were biologically active cells and greatly contributed to pregnancy physiology. Since preeclampsia is considered to be a "trophoblastic cell disease" and distribution of some enzymes changed in preeclamptic villous trophoblasts, and since chorion laeve trophoblasts originate from the same stem cells as villous trophoblasts and play significant roles in maintaining normal pregnancy, it is natural to assume that chorion laeve trophoblasts may also exhibit enzyme distribution changes in preeclampsia, similar to villous trophoblasts. The present study was an effort to clarify this issue. We enzyme-histochemically demonstrated ADP-degrading activity and CCO activity in normal term or near term human chorion laeve trophoblasts, using the lead nitrate (Novikoff and Goldfischer, 1961) and diaminobenzidine (Seligman et al. 1968) methods, respectively. We also compared them with those from preeclamptic chorion laeve trophoblasts.

\section{MATERIALS AND METHODS}

The subjects were ten Japanese pregnant women at term or near term; they were classified into the following two groups: (1), women at 34-39 $(35.8 \pm 2.2$, mean \pm sd.) weeks of gestation without evidence of preeclampsia (gestational age-matched control, $\mathrm{n}=5$ ); and (2), patients with severe preeclampsia at 34-39 (36.0 2 2.0) weeks of gestation with a maternal blood pressure $\geq 160 / 110$ $\mathrm{mmHg}$ on at least two separate occasions and a urinary protein level of $\geq 300 \mathrm{mg} / \mathrm{dl}$ (preeclampsia, $\mathrm{n}=5$ ). After obtaining informed consent, pieces of placental tissue and reflected chorioamnion were obtained within $3 \mathrm{~min}$ of placental delivery. Placental tissues were taken from the central part of the maternal surface, and fetal membranes from three different sites $5 \mathrm{~cm}$ from the placental margin. These specimens were fixed in various concentrations of glutaraldehyde in a cacodylate buffer $(0.1$ $\mathrm{M}, \mathrm{pH}$ 7.4) (2.0\% for villous trophoblastic ADPdegrading activity, $1.0 \%$ for $\mathrm{CCO}$, and $0.5 \%$ for chorion laeve trophoblastic ADP-degrading activity) for 30 mins at $4^{\circ} \mathrm{C}$. Ultrastructural enzyme-histochemistry for ADP-degrading activity and CCO were performed, as previously reported by our group (Matsubara et al. 1997a,b; 2000). In brief, specimens were sectioned $(40 \mu \mathrm{m})$ on a freezing microtome and incubated for $30 \mathrm{~min}$ for ADPdegrading activity, or $120 \mathrm{~min}$ for $\mathrm{CCO}$ at $37^{\circ} \mathrm{C}$ in a corresponding reaction medium: a lead nitrate medium (2.0 mM ADP, $80 \mathrm{mM}$ tris-maleate buffer (pH 7.4), $5.0 \mathrm{mM}$ manganese chloride, $3.6 \mathrm{mM}$ lead nitrate) (Novikoff and Goldfischer 1961) for ADP-degrading activity; and a diaminobenzidine medium $(1 \mathrm{mg} / \mathrm{ml}$ cytochrome c, $100 \mathrm{mM}$ phosphate buffer ( $\mathrm{pH} 7.4$ ), $0.5 \mathrm{mg} / \mathrm{ml}$ diaminobenzidine, $0.1 \mathrm{mg} / \mathrm{ml}$ catalase) (Seligman et al. 1968) for 
CCO. To confirm the specific detection of each enzyme activity, a full series of cytochemical control experiments were performed as previously described (Matsubara et al. 1997a,b). Sections were postfixed in $1.0 \%$ buffered osmium tetroxide for 60 mins, dehydrated and embedded in epoxy resin. Ultrathin sections were prepared, lightly stained with uranyl acetate and lead citrate, and observed under a transmission electron microscope.

\section{RESULTS}

Figure 1 shows the subcellular localization of villous trophoblastic ADP-degrading activity without (a) and with (b) preeclampsia, and CCO without (c) and with (d) preeclampsia. Precipitates indicative of ADP-degrading activity were markedly visible on the syncytial microvillous membranes in normal placentas without preeclampsia (Fig. 1a), but they
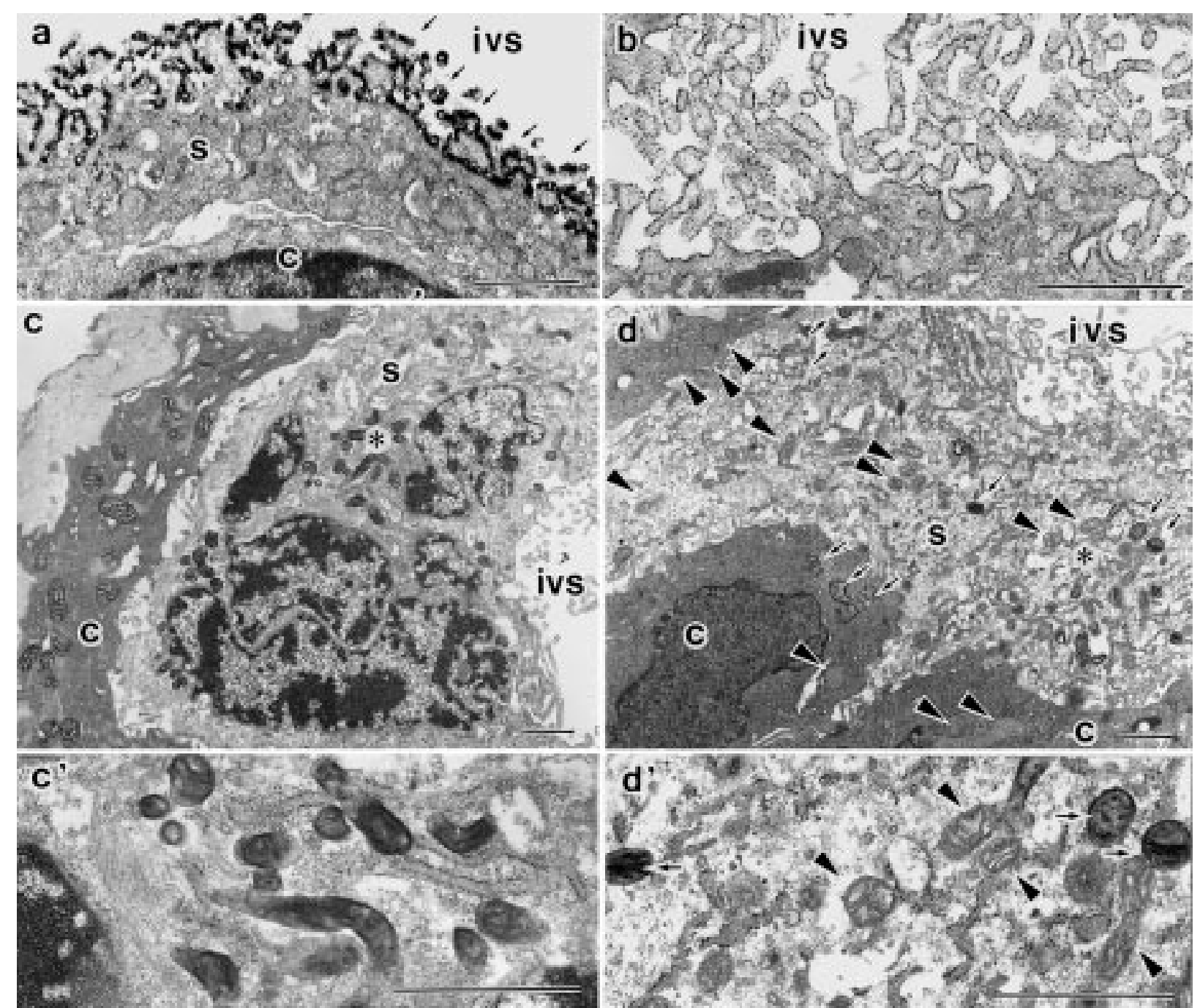

Fig. 1 - Villous trophoblasts. ADP-degrading activity without (a) and with (b) preeclampsia, and cytochrome c oxidase (CCO) without (c, c') and with (d, d') preeclampsia. Precipitates indicative of ADP-degrading activity were visible on the microvillous membranes of the syncytiotrophoblasts in normal placenta without preeclampsia (arrows) (a), and were less dense in a preeclamptic villous syncytium (b). While labeling for $\mathrm{CCO}$ was visible in almost all mitochondria in normal villous trophoblasts (c), in preeclampsia, CCO-positive (arrows) and -negative (arrowheads) mitochondria were observed (d). The areas marked by asterisks in (c) and (d) are shown in (c') and (d'), respectively, at a higher magnification. Co-localization of CCO-positive (arrows) and -negative (arrowheads) mitochondria was evident in preeclamptic villous syncytiotrophoblasts (d'). Bar=1 $\mu \mathrm{m}$. s: syncytiotrophoblast, c: cytotrophoblast, ivs: intervillous space. 
were less dense in preeclamptic placentas (Fig. 1b). As for $\mathrm{CCO}$, in normal villous trophoblasts, almost all mitochondria exhibited positive $\mathrm{CCO}$-staining (Fig. 2c,c'), while in preeclampsia, both CCO-positive and CCO-negative mitochondria co-existed (Fig. $2 \mathrm{~d}$ d').

Figure 2 shows the ultrastructural localization of chorion laeve trophoblastic ADP-degrading activity without (a) and with (b) preeclampsia, and $\mathrm{CCO}$ without (c) and with (d) preeclampsia. Laeve trophoblasts were oval-or polygonal-shaped, and had some microvilli. They were connected to each other by desmosomes, which were useful to distinguish them as laeve trophoblasts. Deposits of ADPdegrading activity were observable on the surface plasma membranes of chorion laeve trophoblasts in normal fetal membranes without preeclampsia (Fig. 2a). In preeclampsia, the intensities and distribution patterns were apparently the same (Fig. $2 \mathrm{~b}$ ) as those in normal pregnancy (Fig. 2a). CCO labelings were visible in the intermembrane spaces and intracristal spaces of all mitochondria of chorion laeve trophoblasts both from normal (Fig. 2c, c') and preeclamptic (Fig. 2d, d') pregnancies. In preeclampsia, CCO-negative mitochondria, which were characteristically observed in preeclamptic villous trophoblasts (Fig. 1d, d'), were not detectable in laeve trophoblasts (Fig. 2d, d'). A series of cytochemical control experiments (i.e., substrate omission, inhibitor addition, section boiling, etc) gave no enzyme labeling (data not shown), ensuring the specific detection of the enzyme activities. Gestational ages did not affect the enzyme distribution, and there were no significant variations in staining pattern in the same group, nor significant differences among sections taken from different sites within the same subjects; thus the observations cited above are believed to be representative findings of various placental/chorioamnion specimens.

\section{DISCUSSION}

We demonstrated previously that two important placental enzymes decreased/changed in their enzyme intensities or distribution patterns in preeclamptic villous trophoblasts compared to normal ones. Firstly, precipitates indicative of ADPdegrading activity on villous syncytial microvillous membranes were less dense in preeclamptic placentas compared to normal ones (Matsubara et al. 1997a). Syncytial microvillous membranes possessed a series of adenine nucleotide-degrading enzymes, such as $\mathrm{Ca}++$ ATPase, ADP-degrading activity, and 5'-nucleotidase (Matsubara et al. 1987), which degraded ATP to ADP, then to AMP, and finally to adenosine, respectively. This microvillous adenine nucleotidase system may modulate platelet adherence to the syncytial surface or platelet aggregation, thus maintaining the fluidity of the maternal blood in the intervillous space (Matsubara et al. 1987). ADP, a potent platelet aggregator, is degraded by placental ADPdegrading activity to AMP (Iioka et al., 1993), which is an anti-platelet aggregator. A decrease in syncytial microvillous ADP-degrading activity may, therefore, lead to accumulation of ADP adjacent to the syncytial microvillous surface, enhancing platelet aggregation in the site and bringing about intervillous thrombus formation, which is frequently observed in preeclamptic placentas (Benirschke and Kaufmann, 2000). Intervillous thrombi may inhibit adequate materno-fetal substance transfer, leading to placental insufficiency. Secondly, preeclamptic villous trophoblasts exhibited a characteristic distribution pattern of CCO. In preeclamptic placentas, $\mathrm{CCO}$-positive and -negative mitochondria co-existed in the villous syncytio- and cytotrophoblasts, while in normal placentas almost all mitochondria showed positive $\mathrm{CCO}$ staining (Matsubara et al. 1997b). We proposed that, in preeclampsia, mitochondrial CCO did not function well, leading to villous trophoblast dysfunction at each cell level, and finally to placental insufficiency in this disease (Matsubara et al. 1997b). Thus, the change/decrease in enzyme activity in preeclamptic villous trophoblasts may be closely associated with the pathogenesis or pathophysiology of preeclampsia. Preeclampsia may, therefore, be a "trophoblastic cell disease".

The present study confirmed the enzyme distribution change characteristic of preeclamptic villous trophoblasts. We did not, however, find a decrease/change in enzyme intensities and distribution pattern of two enzymes in preeclamptic chorion laeve trophoblasts. The distribution patterns of ADP-degrading activity and CCO in preeclampsia differed between villous trophoblasts and laeve trophoblasts. We do not know the reason for this phe- 

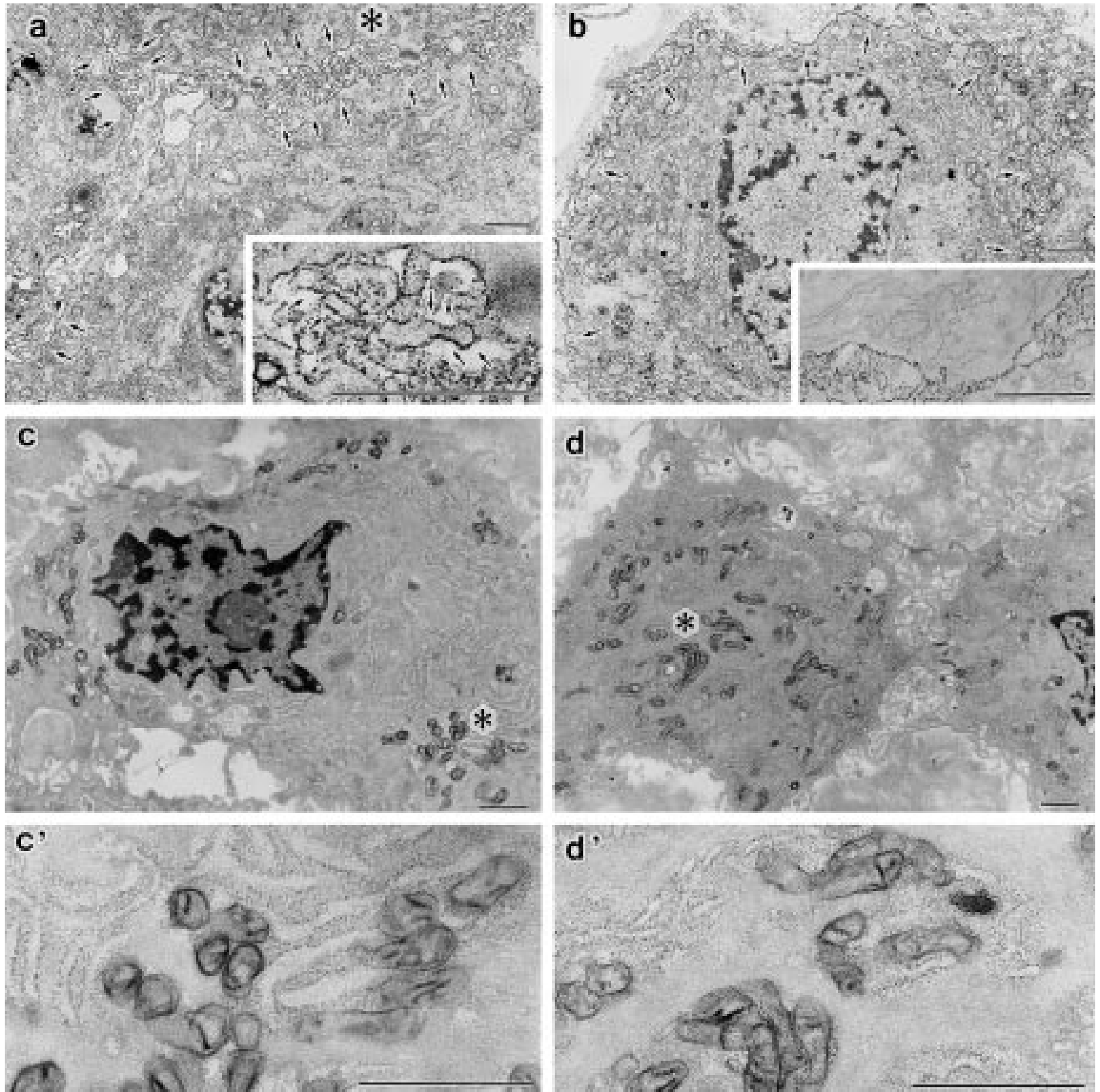

Fig. 2 - Chorion laeve trophoblasts. ADP-degrading activity in normal (a) and preeclamptic (b) pregnancy, and CCO without (c, $\left.c^{\prime}\right)$ and with (d, d') preeclampsia. Deposits indicative of ADP-degrading activity (arrows) were visible on surface microvillous membranes of chorion laeve trophoblasts from normal (a) and preeclamptic (b) pregnancy. The area marked by an asterisk in (a) is shown in the inset at a higher magnification. Deposits (arrows) for ADP-degrading activity were visible on the surface plasma membranes. The inset of (b) is an electron micrograph of an unstained specimen from another preeclamptic subject. Precipitates were clearly visible on the plasma membranes of two adjacent laeve trophoblasts. Intensities of deposits were apparently the same between normal (a) and preeclamptic (b) subjects. The figures (c, $\left.c^{\prime}\right)$ and $\left(d, d^{\prime}\right)$ indicate CCO activity. CCO labeling was visible in almost all mitochondria both in normal $\left(c, c^{\prime}\right)$ and preeclamptic $\left(d, d^{\prime}\right)$ chorion laeve trophoblasts. The areas marked by asterisks in (c) and (d) are shown in (c') and (d'), respectively, at a higher magnification. Leave trophoblasts from preeclampsia did not have any CCO-negative mitochondria. $\mathrm{Bar}=1 \mu \mathrm{m}$.

nomenon. One possible reason, however, may be a lack of, or lower degree of, laeve trophoblastic hypoxia compared to villous trophoblastic hypoxia. In preeclamptic placentas, physiological dilatation 
of placental bed spiral arteries, characteristic of normal pregnancy, does not occur. The lack of placenta vessel dilatation reduces blood influx into the intervillous space, resulting in intervillous hypoxia in preeclampsia (Redman 1991). As villous trophoblasts depend on intervillous maternal blood for their oxygen, they may suffer from hypoxia in preeclampsia (Redman 1991; Widschwendter et al. 1998), which may lead to trophoblast cell dysfunction, and finally to decreased enzyme activities. Chorion laeve trophoblasts, on the other hand, receive oxygen from underlying maternal parietal decidual vessels. In these vessels, unlike those of the placental bed, physiological vessel dilatation does not occur even in normal pregnancy, and thus parietal decidual vessel blood flow may remain intact in preeclampsia. Hypoxia in parietal decidual vessel blood (oxygen supplier for laeve trophoblasts), may, therefore, be absent, or at least less severe, than in intervillous blood (oxygen supplier for villous trophoblasts), though direct measurement of the oxygen content at these sites is impossible. Thus, the oxygen supply to laeve trophoblasts may remain intact, or may be less disturbed, thus maintaining enzyme activities even in preeclampsia. This is hypothetical, although reasonable. Whatever the reason for the lack of decreased enzyme activities in preeclamptic laeve trophoblasts, the present enzyme-histochemical study indicated that there may be no, or at least less severe, abnormalities in the enzyme activities of chorion laeve trophoblasts compared to villous trophoblasts.

One possible cause of preeclampsia is a mitochondrial disorder (Widschwendter et al. 1998). Torbergsen et al. (1989) observed a high incidence of preeclampsia in a family with mitochondrial dysfunction. Since then, epidemiological (Berkowitz et al. 1990), genetic (Folgerø et al. 1996), and biochemical (Furui et al. 1994) studies have strongly suggested a close association between preeclampsia and placental mitochondrial dysfunction. Folgerø et al. (1996) detected mutations in mitochondrial tRNA genes in families with a high occurrence of preeclampsia. We also observed CCO-negative mitochondria in preeclamptic villous trophoblasts (Matsubara et al. 1997b), which was reconfirmed by the present study. In the present study, however, CCO-negative mitochondria were not observed in preeclamptic laeve trophoblasts. Cells, if they have syncytial features, are more likely to be involved in mitochondrial abnormalities (Clarke 1990). Villous trophoblasts consist of two populations; syncytiotrophoblasts and cytotrophoblasts. In vitro experiments and in vivo observations showed that villous cytotrophoblasts fuse to each other or to adjacent syncytiotrophoblasts (Benirschke and Kaufmann 2000), and thus have the capacity to form the syncytium. Chorion laeve trophoblasts, on the other hand, do not have the capacity to form syncytia (Benirschke and Kaufmann 2000), which may account for their lack of change in mitochondrial $\mathrm{CCO}$ in preeclampsia.

Physiological roles of these two enzymes in fetal membrane biology are not known. The chorion laeve trophoblast surface plasma membranes had $\mathrm{Ca}++$ ATPase and 5'-nucleotidase (Matsubara et al. 1994), and ADP-degrading activity (the present study); thus they have a complete adenine nucleotidase system (degrading ATP $\rightarrow$ ADP $\rightarrow$ AMP $\rightarrow$ adenosine), similar to villous syncytial microvilli (Matsubara et al. 1987; 1997a). While the villous adenine nucleotidase system may maintain the blood fluidity of the intervillous space as cited previously, laeve trophoblastic adenine nucleotidases, including ADP-degrading activity, may have other roles, as laeve trophoblasts do not have contact with the maternal blood. Cell-to-cell signal transduction, a well known function of adenosine in other tissues (Fishman et al. 2000), may be one possible role, although direct evidence is lacking. $\mathrm{CCO}$, the important enzyme of the mitochondrial respiratory chain, is required for cell respiration and energy supply within cells (Alberts et al. 1994). As cited previously, laeve trophoblasts are metabolically active cells, which demand a large energy supply for their various functions/metabolism. CCO may, therefore, fuel laeve trophoblasts with energy. Though details are obscure, these two enzymes may play significant roles in fetal membrane physiology, and it has now become clear that cytochemically detectable enzyme activities remain intact in preeclamptic laeve trophoblasts.

In conclusion, we demonstrated a subcellular localization of ADP-degrading activity and CCO in human fetal membrane chorion laeve trophoblasts from normal and preeclamptic pregnancies. ADPdegrading activity was present on the surface plasma membranes of laeve trophoblasts, and CCO labeling was visible in all laeve trophoblastic mitochondria both in normal and preeclamptic subjects. 
A decrease in ADP-degrading activity and the presence of CCO-negative mitochondria, which were characteristic of preeclamptic villous trophoblasts, were not observed in preeclamptic laeve trophoblasts. In preeclampsia, there may be no, or at least less severe, abnormalities in laeve trophoblast enzyme functions, compared to those of villous trophoblasts. Preeclampsia, therefore, may be a "villous" trophoblastic disease, but not a "laeve" trophoblastic disease, solely from an enzyme-histochemical viewpoint. Although we elucidated some of the features of chorion laeve trophoblasts in normal and pathological (preeclampsia) conditions, further studies using methods other than enzymehistochemistry are necessary to elucidate the roles these two enzymes play in fetal membrane physiology in both conditions.

\section{REFERENCES}

Alberts B., Bray B., Lewis J., Raff M., Roberts K., and Watson J.D.: Energy conversion: mitochondria and chloroplasts. In Molecular Biology of the Cell. (Eds. Alberts B., Bray B., Lewis J., Raff M., Roberts K. and Watson J.D.) Garland, New York, pp. 653-720, 1994

Benirschke K. and Kaufmann P.: Pathology of the human placenta, Springer-Verlag, New York, 2000.

Berkowitz K., Montaegudo A., Marks F,. Jackson U. and Baxi L.: Mitochondrial myopathy and pre-eclampsia associated with pregnancy. Am. J. Obstet. Gynecol. 162, 146-147, 1990.

Clarke A.: Mitochondrial genome: defects, disease, and evolution. J. Med. Genet. 27, 451-456, 1990.

Cunningham F.G., MacDonald P.C., Gant N.F., Leveno K.J., Gilstrap L.C., Hankins G.D.V., and Clark S.L.: Hypertensive disorders in pregnancy. In Williams Obstetrics, $20^{\text {th }}$ edn. (Eds. Cunningham, F.G., MacDonald, P.C., Gant, N.F., Leveno, K.J., Gilstrap, L.C., Hankins, G.D.V., and Clark, S.L.) Appleton\&Lange, Connecticut, pp. 693-744, 1997.

Fishman P., Bar-Yehuda S., Farbstein T., Barer F., and Ohana G.: Adenosine acts as a chemoprotective agent by stimulating G-CSF production: a role for A1 and A3 adenosine receptors. J. Cell Physiol. 183, 393-398, 2000.

Folgerø T., Storbakk N., Torbergsen T., and Øian P.: Mutations in mitochondrial transfer ribonucleic acid genes in preeclampsia. Am. J. Obstet. Gynecol. 174, 1626-1630, 1996.

Furui T., Kurauchi O,. Tanaka M., Mizutani S., Ozawa T., and Tomoda Y.: Decrease in cytochrome c oxidase and cytochrome oxidase subunit I messenger RNA levels in preeclamptic pregnancies. Obstet. Gynecol. 84, 283-288, 1994.

Germain A.M., Smith J., Casey M.L., and MacDonald P.C.: Human fetal membrane contribution to the prevention of par- turition: uterotonin degradation. J. Clin. Endocrinol. Metab. 78, 463-470, 1994.

Iioka H., Akada S., Sakamoto Y., Shimamoto T., Yamada Y, Sakamoto Y., Moriyama S.I., and Ichijo M.: Properties of ADP-degrading activity of human syncytial brush border membrane vesicles. Placenta 14, 333-339, 1993.

Matsubara S., and Sato I.: Enzyme-histochemically detectable glucose-6-phosphatase is present in chorion laeve trophoblasts. Histochem. Cell Biol. 113, 363-368, 2000.

Matsubara S., Tamada T., Kurahashi K., and Saito T.: Ultracytochemical localization of adenosine nucleotidase activities in the human term placenta, with special reference to 5'-nucleotidase activity. Acta Histochem. Cytochem. 20, 409-419, 1987.

Matsubara S., Sayama M., Sato I., and Tamada T.: Enzymecytochemistry of human term chorion laeve at term: enzyme localization on the chorionic trophoblast. Asia-Oceania J. Obstet. Gynecol. 20, 217-222, 1994.

Matsubara S., Sato I., and Saito T.: Ultrastructural localization and cytochemical characteristics of human placental ADP-degrading activity in normal and preeclamptic pregnancy. Trophoblast Research 9, 121-129, 1997a.

Matsubara S., Minakami H., Sato I., and Saito T.: Decrease in cytochrome c oxidase activity detected cytochemically in the placental trophoblast of patients with pre-eclampsia. Placenta $18,255-259,1997 \mathrm{~b}$

Matsubara S., Minakami H., Takayama T., and Sato I.: Cellular and subcellular localization of nicotinamide adenine dinucleotide diaphorase activity in the human placenta. J. Obstet. Gynecol. Res. 23, 133-138, 1997c.

Matsubara S., Takizawa T., and Sato I.: Enzyme-histochemistry on the human placental trophoblasts: the effect of fixation delay on the enzyme activity. Histochem. Cell Biol. 113, 287-292, 2000.

Novikoff A.B., and Goldfischer S.: Nucleotide diphosphatase activity in the Golgi apparatus and its usefulness for cytochemical study. Proc. Natl. Acad. Sci. USA. 47, 802-810, 1961.

Poisner A.M., Wood G.W., Poisner R., and Inagami T.: Localization of renin in trophoblasts in human chorion laeve at term pregnancy. Endocrinology 109, 1150-1155, 1981.

Redman C.W.G.: Current topic: preeclampsia and the placenta. Placenta 12, 301-308, 1991

Sangha R.K., Walton J.C., Ensor C.M., Tai H.H., and Challis R.G.: Immunohistochemical localization, messenger ribonucleic acid abundance, and activity of 15-hydroxyprostaglandin dehydrogenase in placenta and fetal membranes during term and preterm labor. J. Clin. Endocrinol. Metab. 78, 982-989, 1994.

Seligman A.M., Karnovsky M.J., Wasserkrug H.L., and Hanker J.S. Nondroplet ultrastructural demonstration of cytochrome oxidase activity with a polymerizing osmiophilic reagent, diaminobenzidine (DAB). J. Cell Biol. 38, 1-14, 1968. 